\title{
Acetabular Component Position Highly Variable in Early Practice
}

\section{Caitlin Barrett ${ }^{1}$, Jillian Glasser ${ }^{1}$, Brooke Barrow ${ }^{2}$, Dioscaris Garcia ${ }^{2}$ and Valentin Antoci ${ }^{1,2 *}$}

${ }^{1}$ University Orthopedics Inc., Providence, Rhode Island

${ }^{2}$ Department of Orthopaedic Surgery, Warren Alpert School of Medicine, Brown University, Providence, Rhode Island

*Corresponding Author: Valentin Antoci, Associate Professor, Department of Orthopaedic Surgery, Brown University, University Orthopedics Inc., East Providence, Rhode Island.
Received: August 11, 2021

Published: August 21, 2021

(C) All rights are reserved by Valentin Antoci., et al.

\begin{abstract}
One factor in choosing an orthopedic fellowship is to increase practice with standard procedures, such as acetabular cup placement. The purpose of this study is to see how adult reconstruction fellowship affects surgical accuracy in practice. This retrospective review compares anteversion/abduction measurements and outcomes of 50 THAs done during fellowship to 50 THAs performed in clinical practice. Abduction and anteversion in fellowship averaged $46.14^{\circ}$ and $23.9^{\circ}$, compared to $45.16^{\circ}$ and $29.86^{\circ}$ in practice. There was no significant difference in complications between groups. These results suggest that fellowship training decreases but does not eliminate the learning curve for acetabular cup placement.
\end{abstract}

Keywords: Total Hip Arthroplasty; Acetabular Cup Placement; Fellowship; Learning Curve

\section{Introduction}

The field of orthopedic surgery has become increasingly specialized over the last few decades, and more surgeons are pursuing fellowships after residency than ever before. According to the American Medical Association (AMA), 87.4\% of orthopedic surgery residents are planning to pursue a fellowship, the highest rate among all surgical specialties [1]. This number is greatly increased from the $76 \%$ of orthopedic surgeons with fellowship training who took their American Board of Orthopaedic Surgery Part II examinations in 2003 [2]. With the increase in volume of trainees looking to specialize, the amount of fellowship positions has significantly increased in all areas, except spine and trauma [3].

While there are many potential reasons why a resident may want to pursue a fellowship, some of the most significant ones are to increase their exposure to more complex cases and to get more practice with standard procedures [4]. The latter could have a specific benefit to adult reconstruction fellows due to the planning and reproducibility needed to position total hip arthroplasty
(THA) components [5]. Additionally, fellowship training has a significant impact on implant selection, and is one of the most influential driving factors of implant choice [6].

In 2015, Almansoori., et al. found that Canadian residencies accurately prepared orthopedic surgeons to enter practice, and fellowship pursuance was not due to poor residency training [7]. However, United States and Canadian orthopedic training programs differ significantly, making direct comparison difficult [8]. Currently in the United States, general surgical (GS) trainees feel that they have insufficient preparation upon the end of their residency, with $38 \%$ of residents in these programs responding "no" or "unsure' when asked if a 5-year GS residency would fully prepare you to practice GS [9]. While little information on this topic exists for the field of orthopedic surgery in America, higher rates of fellowship pursuance could suggest a similar sentiment. This could suggest that the current 5-year residency program is not adequate, and without amending this system, pursuing a fellowship is strongly recommended [1]. 


\section{Purpose of the Study}

The purpose of this study is to see what effect adult reconstruction fellowship experiences have on surgical accuracy in practice. While fellowship training can help provide more repetition and exposure to complex cases, we expect that there is still a significant learning curve when starting a new practice. Acetabular component position is the most difficult part of a total hip arthroplasty, with high variability reported using standard technique and alignment.

\section{Objective of the Study}

The objective of this study was to evaluate surgical accuracy of placing the acetabular component during fellowship and measure any continued change through the first year in practice. Our null hypothesis is that fellowship should provide enough training wherein there is no learning curve and no change in variability when entering practice.

\section{Methods}

This is an IRB-approved retrospective chart review comparing the outcomes of 50 total hip arthroplasties done during fellowship under supervision of an expert surgeon to 50 total hip arthroplasties performed post-fellowship early in clinical practice. The patients were matched in terms of demographics, patient age, and comorbidity status. All cases were performed with a minimally invasive posterolateral approach to the hip. Success was assessed through the analysis of acetabular cup positioning with abduction and anteversion measurements calculated for each patient. Radiographic images for each case were processed with BrainLab TraumaCad (Westchster, IL), and anteversion was assessed algorithmically on the AP pelvis. Patient charts were retrospectively reviewed for patient complications, dislocation, and readmission.

All statistics were evaluated using STATA and Microsoft Excel Analysis toolpak with significance set at $\mathrm{p}<0.05$. The 50-patient cohort was chosen based on appropriate power calculation of 0.8 . This is a level III retrospective cohort study.

\section{Results}

For the 50 cases in fellowship under the supervision of an expert surgeon, the average abduction measurement was 46.14 degrees and the average anteversion measurement was 23.9 degrees. Variance for both measurements were 10.54 degrees. Figure 1 and
2 show the average anteversion and abduction over the course of the 50 surgeries. Variation between cases remained rather steady over time.

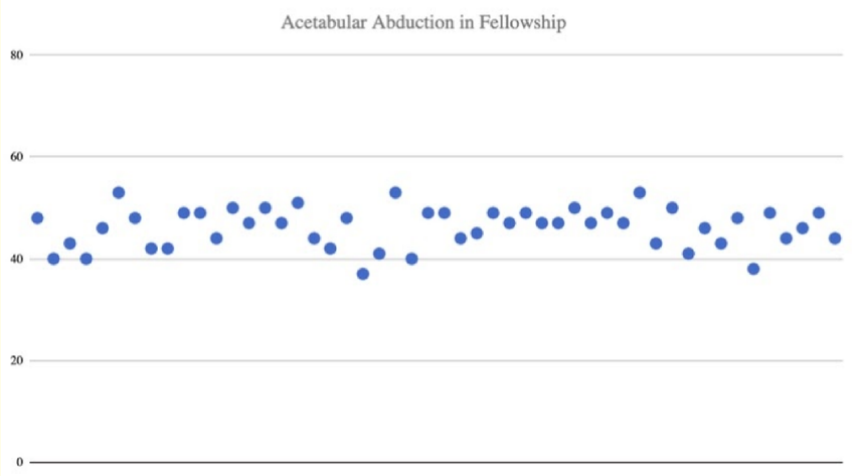

Figure 1: Average abduction (in degrees) during the 50 cases during fellowship.

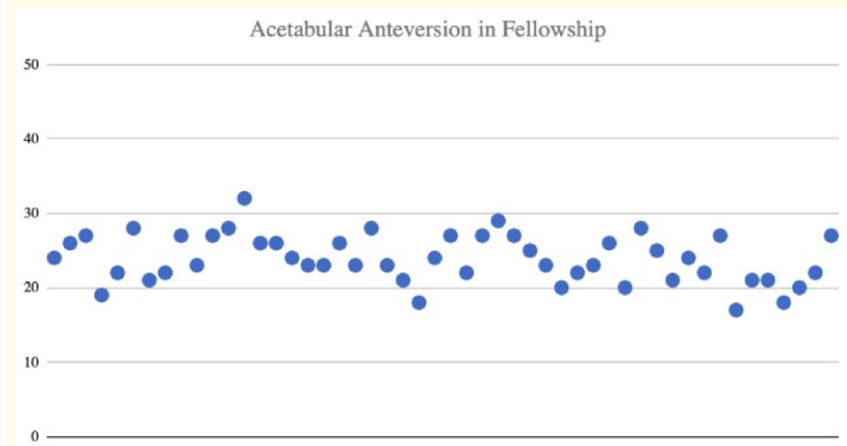

Figure 2: Average anteversion (in degrees) during the 50 cases during fellowship.

For the 50 cases in practice, the average abduction measurement was 45.16 degrees and the average anteversion measurement was 29.86 degrees. The average abduction in fellowship and practice only differed by 0.01 degree, while anteversion was significantly higher in practice at 29.86 degrees, compared to 23.9 degrees in fellowship. Variance for abduction measurements in 
practice was 58.87 degrees, while variance for anteversion measurements was 38.08 degrees. Both variance measurements were increases from cases done during fellowship training. Figure 3 and 4 show the average anteversion and abduction over the course of the 50 surgeries in early practice. Abduction variation was highest among the first 20 cases and improved afterwards. However, they never approached the reproducibility achieved in fellowship. The trend in the anteversion measurements in practice showed large variation among all 50 cases.

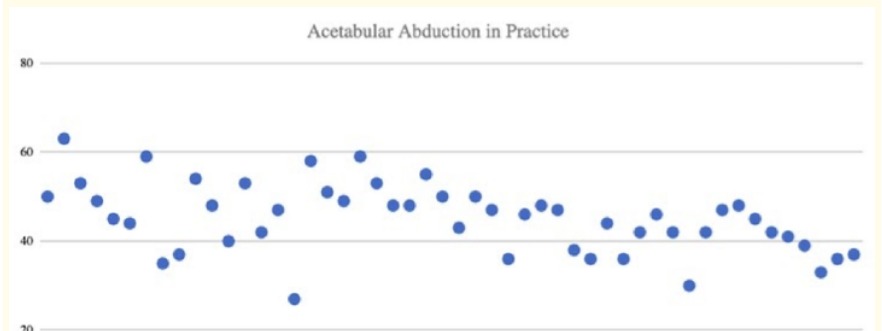

Figure 3: Average abduction (in degrees) during the 50 cases in early practice.

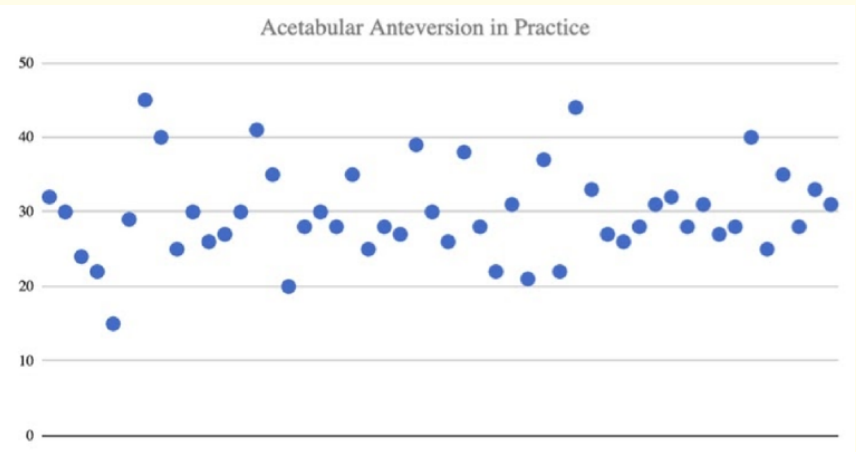

Figure 4: Average anteversion (in degrees) during the 50 cases in early practice.

No dislocations were observed in either cohort. The rate of readmission was $2.45 \%$ mainly for lower extremity swelling, pain, and placement issues. When comparing the fellowship cohort and practice cohort, no significant change in rate of dislocations, readmissions, or other complications were seen in either patient population at 1 year after surgery.

\section{Discussion}

The results of our study indicate that fellowship training decreases but does not eliminate the learning curve for THA acetabular cup placement in community practice. Our data reveals a learning curve of at least 20 patients in early practice, but variability continues to slowly improve way beyond that. Measurements for anteversion and the variance for abduction and anteversion were all higher in practice than in fellowship. The variation decreased for all cases following the initial 20 patient learning curve. Our results indicate that acetabular component positioning is much more reproducible in the presence of an expert surgeon such as in fellowship. While fellowship training increases exposure to complex cases, independent practice still requires caution and continuous optimization.

The current literature suggests an even longer learning curve than found in our study. Lee., et al. examined the presence of a learning curve of about 40 cases for acetabular cup positioning in total hip arthroplasty [10]. This study evaluated the outcomes of the first 100 total hip arthroplasty procedures of two surgeons after they completed a year of fellowship at the same institution. The learning curve cumulative summation test signaled competency after 43 procedures for surgeon 1 and after 35 procedures for surgeon 2, both higher than the 20 cases in our study. The surgeons maintained competency for the remainder of the 100 cases, similar to the decrease in variation of measurements following the learning curve in our study.

Similarly, a 2018 single-surgeon study by Van Den Eeden., et al. examined the learning curve associated with performing the direct anterior approach for primary THA [11]. After reviewing operative parameters, overall complications and radiological component placement over the course of the first 200 cases utilizing the direct anterior approach, a much higher learning curve of 100 cases was reported. However, the surgeon was not specified to be directly out of fellowship and utilized a different approach than that of our study. This study confirms the presence of a learning curve across multiple surgical approaches for THA; however, further investigation into the impact of fellowship training on the direct anterior approach is needed for a direct comparison to our study. 
A 2017 study by Kim., et al. investigated the impact of level of surgical experience on outcomes of THA [12]. This study does not report an actual number for a learning curve associated with center of rotation (COR) position and acetabular cup placement; however, the data shows a clear effect of level of surgical experience on postoperative COR position and acetabular cup inclination. Greater experience was associated with more accurate and consistent cup placement. Our finding that acetabular component positioning is more reproducible during fellowship suggests that just operating with an experienced surgeon can improve accuracy and consistency.

While past studies have indicated the presence of a learning curve when performing total hip arthroplasty, our findings are notable because they suggest a significantly shorter learning curve. The current literature suggests that learning curves may be affected by several factors including the type of surgical approach used. Insight into the type of fellowship training received by each surgeon and the subsequent effect on their personal learning curve is needed to understand the different lengths of learning curves.

Limitations of this study include a relatively small sample size of 50 cases during fellowship and 50 cases post-fellowship, even though appropriately powered. Furthermore, our study was limited to cases performed by one surgeon and one approach. Learning curves may be influenced by several factors, including the institution of fellowship-training. Consequently, the findings of our study may not be generalizable to the broader population of surgeons performing total joint arthroplasty.

\section{Conclusion}

The learning curve for acetabular cup placement of only 20 patients reported in this study is much lower than the average learning curve reported in the literature. Our results suggest that fellowship significantly improves judgement and provides the opportunity to continuously adjust technique, leading to better overall outcomes in post-fellowship practice. Further investigations comparing the outcomes of cases performed during fellowship and early in clinical practice on a larger scale and across multiple institutions are needed to further elucidate the impact of fellowship training on total joint arthroplasty skill once surgeons are in community practice. However, this study provides valuable insight into the beneficial impact of fellowship training on later clinical outcomes and presents a novel finding of a shorter learning curve. This information is important for those making decisions about pursuing fellowship, those in fellowship, and those beginning community practice.

\section{Acknowledgements and Disclosures}

The authors did not receive grant support or research funding to complete this study and do not have any proprietary interests in the materials described in the article. All author disclosures are listed on attached disclosure forms.

\section{Bibliography}

1. American Medical Association. FREIDA Online graduates' career plans search: Orthopaedic Surgery (2013).

2. Horst PK., et al. "Graduates of orthopaedic residency training are increasingly subspecialized a review of the American board of orthopaedic surgery part II database". Journal of Bone and Joint Surgery - American Volume (2014).

3. Ruddell JH., et al. "Trends in the Orthopaedic Surgery Subspecialty Fellowship Match: Assessment of 2010 to 2017 Applicant and Program Data". The Journal of Bone and Joint Surgery American Volume 100.21 (2018): e139.

4. Wylie JD. "Pearls: Getting the Most Out of Your Fellowship Year". Clinical Orthopaedics and Related Research 477.5 (2019): 958-959.

5. Colombi A., et al. "Total hip arthroplasty planning". EFORT Open Reviews 4.11 (2019): 626-632.

6. Moss L., et al. "Current Practice Patterns of Fellowship-Trained Arthroplasty Surgeons: Has the Influence of Fellowship Training Been Undervalued?" Journal of Arthroplasty 34.5 (2019): 1003-1007.

7. Almansoori KA and Clark M. "Increasing Trends in Orthopedic Fellowships Are Not due to Inadequate Residency Training". Education Research International (2015): 1-9.

8. Syed S., et al. "A brief comparison of orthopaedic training in English-speaking countries". Annals of the Royal College of Surgeons of England 91.3 (2009): 226-231.

9. Coleman JJ., et al. "Early subspecialization and perceived competence in surgical training: Are residents ready?" Journal of the American College of Surgeons 216.4 (2013): 764-771. 
10. Lee YK., et al. "Learning curve of acetabular cup positioning in total hip arthroplasty using a cumulative summation test for learning curve (LC-CUSUM)". Journal of Arthroplasty 29.3 (2014): 586-589.

11. Van Den Eeden Y and Van Den Eeden F. "Learning curve of direct anterior total hip arthroplasty: a single surgeon experience". Acta orthopaedica Belgica 84.3 (2018): 321-330.

12. Kim S-C., et al. "Level of surgical experience is associated with change in hip center of rotation following cementless total hip arthroplasty: A radiographic assessment". PLOS ONE 12.5 (2017): e0178300.

Volume 4 Issue 9 September 2021

(C) All rights are reserved by Valentin Antoci., et al. 